\title{
Smart Process Combination for Aluminum/Plastic Hybrid Components
}

\author{
André Albert'), Wolfgang Zorn ${ }^{1}$, Markus Layer ${ }^{2)}$, Welf-Guntram Drossel ${ }^{1)}$, Dirk Landgrebe ${ }^{1)}$, Lothar \\ Kroll2), Wolfgang Nendel ${ }^{2)}$ \\ 1) Fraunhofer Institute for Machine Tools and Forming Technologies, Reichenhainer Straße 88, \\ 09126 Chemnitz, Germany \\ 2) Department of Lightweight Structures and Polymer Technology, slk@mb.tu-chemnitz.de, \\ Chemnitz University of Technology, Reichenhainer Straße 31/33, 09126 Chemnitz, Germany
}

\section{Keywords}

metal/plastic, hybrid components, production engineering, lightweight construction, active components

\begin{abstract}
The research on lightweight construction increasingly gains in importance, especially for the automotive industry. New lightweight components ensure the necessary stability of car body parts on the one hand. On the other hand they are supposed to allow a low priced production. Hence, aluminum or magnesium alloys have quite a large share in production engineering. During the last years, research mainly addressed metal/plastic compounds. Weight reduction as well as the capability of producing complex structures are only some of the benefits of this technology. Furthermore, additional functionality can be integrated or functional tasks can be distributed: The metal ensures stiffness and realizes the technical connection to the car body by means of welding, while the plastic enables the insertion of special elements for the joining or assembly process. This paper presents two approaches of realizing a combined process to produce aluminum/plastic-hybrid structures. In a first approach, an active tool is presented to realize the sheet based process. The second approach focusses on the tube-based process and presents the topical state of research within the Federal Cluster of Excellence EXC 1075 "Merge Technologies for Multifunctional Lightweight Structures".
\end{abstract}

\section{Introduction}

Lightweight solutions for the automotive industry are today, more than ever, in demand. In addition to the use of materials like aluminum, magnesium, high-strength or even ultra-high-strength steels, composite designs of synthetic materials and metals are increasingly being used for weight reduction and functional integration purposes [1]. In recent years, hybrid components have been especially employed in cars. For the most part these components consist of a thin-walled metal structure in conjunction with a synthetic reinforcement (Fig. 1) [2]. With tube-based hybrid components, the weight-reduction potential of flangeless metallic hollow sections can be combined with highly complex injection molded synthetic elements. These components have so far been used by Mercedes-Benz as cockpit cross-members or as car front-ends. They are manufactured in a combined process of hydroforming (HF) and injection molding inside a single tool. The metal profile is initially shaped by hydroforming. Then, the injection molding process is carried out while maintaining the internal pressure in order to prevent the hollow profile from collapsing. 




Figure 1: Hybrid Metal-Plastics-Frontend of an Audi TT [3] und pedal bearing block of a Mercedes C-Klasse [4]

The realization of such hybrid structures is currently carried out either by the subsequent joining of each part (Post Molding Assembly, PMA) or by an injection molded integrated joining (Injection Molding Assembly, IMA) [5]. In PMA, the individual components are produced in separate processes and then connected to one another. In IMA, the metal structure is first produced; the synthetic structure is realized afterwards within an injection tool. In most cases, the metal components must be provided with a coupling agent before the joining. A similar procedure exists for fiber composites instead of metal [6].

In order to simplify this process chain further, the company Corus has developed the so-called Polymer Injection Molding (PIM) [7]. In this case, the metallic sheet coated with the coupling agent is inserted into the injection molding tool and upon closing the semi-finished product is preformed mechanically. The subsequent injection molding process can be used for the forming of the metal component. This process allows for a one-stage production of hybrid components, provided that the metal component has a very simple shape.

Beyond the state of industrial applications, research has been done concerning the production of hybrid components using molten plastic as a medium for the forming [8] or considering the pressuremedium-based production of a hybrid metal-plastic composite component with plastic melt as a printing medium [9].

The process chain for the production of hybrid components that has been shown so far is currently complex and cost-intensive. The main reason for this is the separation of metal-forming and injection molding as well as the use of a cost-intensive chemical coupling agent. The aim of the Federal Cluster of Excellence MERGE was to shorten the process chain and thereby significantly reduce production costs. The production process was supposed to be carried out in so-called in-situ processes in a single production step. There, primary forming (injection molding), forming (deep-drawing, HF) and joining are to be carried out in one tool in one process (Figure 2 right). For the production of sheetbased hybrid components, the polymer injection molding process previously applied only to injection molding machines was supposed to be transferred to deep-drawing machines. By not transferring the metal forming process to the injection molding machine, but instead integrating the injection molding process into the press plant, the production of considerably more complex deep-drawn parts was to be rendered possible.

The HF-injection molding for the production of tube-based hybrid components was to be developed further in MERGE. The aim was to replace chemical coupling agents by surface-structuring the tubes as well as using gaseous media for internal high-pressure forming (Figure 2 left). 


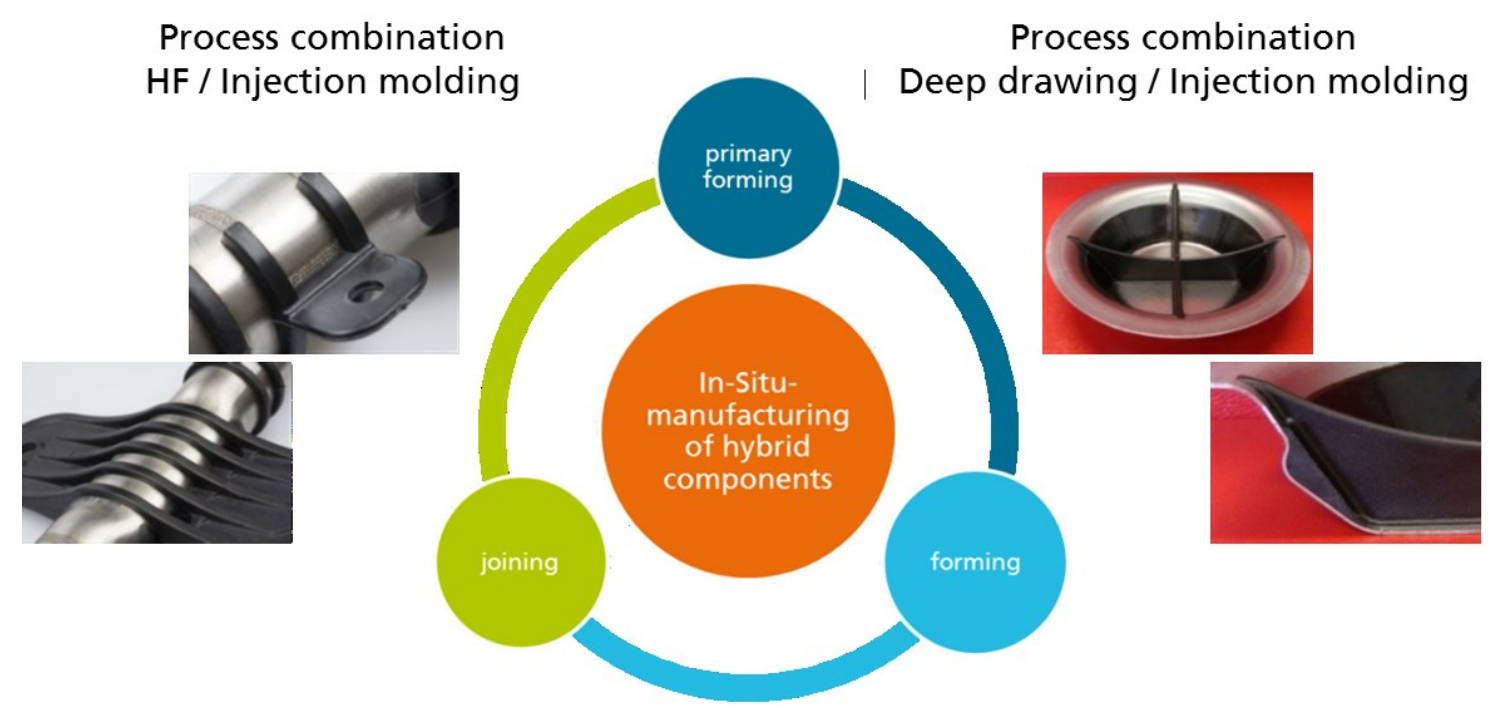

Figure 2: In-Situ-Processes for manufacturing of Metal-Plastics-Hybrid-Components

\section{Sheet based process: combination of sheet metal forming and injection molding}

For the sheet-based hybrid process, a corresponding tool was developed in recent research activities. This allows for a largely independent application of the separate process components (deep-drawing and injection molding with additional active media-based forming). The tool is shown as a CAD model in Figure 3. It consists of an upper tool with a die, as well as a lower tool including the punch, the blank holder as well as the hot channel with connection to the injection molding unit. With respect to the flowability of the plastic on the one hand, as well as the cycle time restrictions on the other hand, the tool is equipped with a fluidic temperature control. For this, the tool components are heated or cooled by means of the integrated ducts with water, whereby a primary importance of the heating is to be considered for investigating the processability.

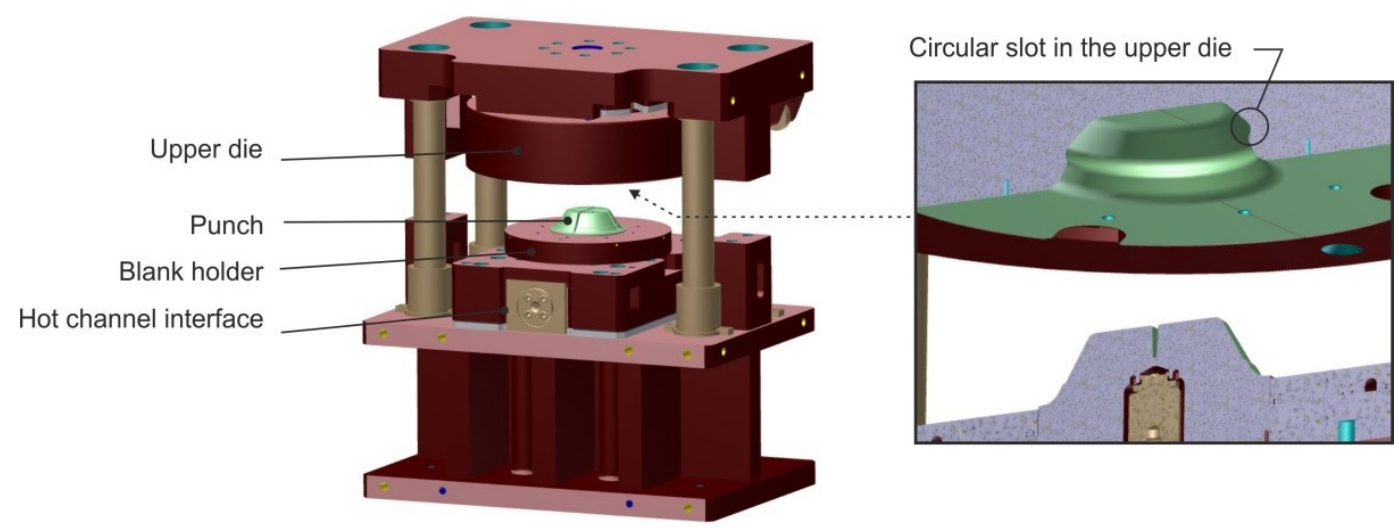

Figure 3: CAD-Illustration of the tool (I.h.s.), detailed illustration of the upper die (r.h.s.)

The punch and its geometry in addition to the counterform to the die realize the inverse for the plastic structure as well, which is injected through the die. To shape the rib structure, the upper part of the punch is divided into four segments in which heating channels are integrated. These are designed in such a way that, on the one hand, a dynamic temperature control near the surface can be achieved. On the other hand there is space for the integration of further elements. For example, heating cartridges can be provided for additional heating or as sensor elements. In this case, a combined sensor was chosen for the purpose of measuring the plastic pressure and temperature in an effective 
manner. In addition, thermocouples (Type K) are used for measuring the internal temperature of the punch. With respect to the all-encompassing temperature control, the blank holder can be both heated and cooled by integrated temperature control channels. Since the channels are provided within a plane, the blank holder can also be produced using the conventional milling process. Seeing that steel has mainly been used as a sheet material in previous research activities, the use of aluminum (EN AW-6016) is to be focused on in a second experimental series in order to be able to investigate additional potential (e.g. weight reduction) of the component. Aluminum and magnesium alloys are currently widely used in industrial production, which have a markedly reduced specific weight compared to steel, but are also much more difficult to form. Comparing the mechanical properties of untreated or aged aluminum and steel, the elongation at break of steel is significantly higher than in aluminum. In the process discussed here, the aluminum blanks without any heat treatment already crack during the forming step, so that subsequent injection molding is no longer possible. Figure 4 shows a direct comparison of the formed metal blanks before the injection of the plastic.

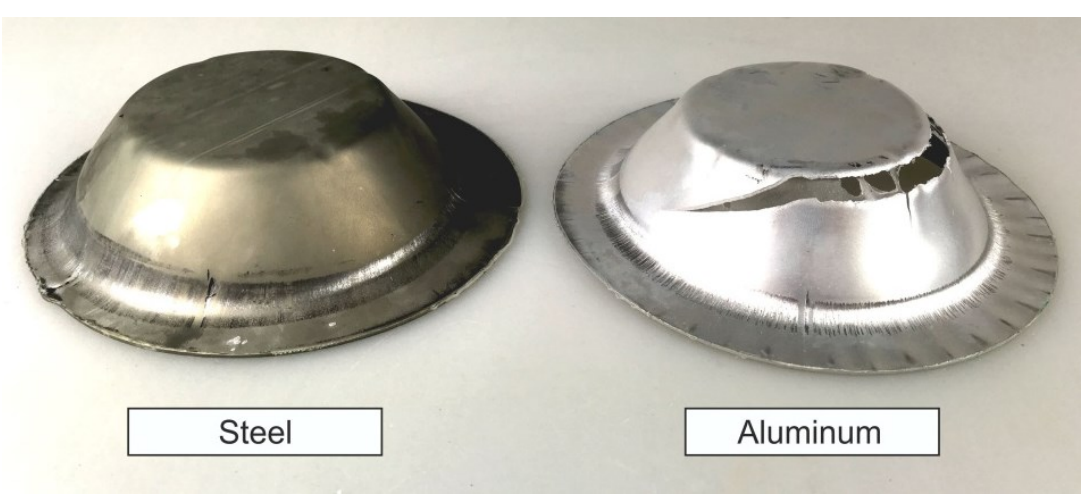

Figure 4: Comparison of deep-drawing results: steel (1.0338) and aluminum (EN AW-6016, with bonding agent)

In practice, semi-finished products based on aluminum are subjected to a pre-set heat treatment in order to increase the strength of the material. The setting of lower forming speeds and the use of several forming steps represent further useful alternatives for optimizing the flow behavior [10]. However, these approaches are only partially flexible in increasing the process reliability during the production of aluminum components at the same cycle time. One further approach is the concept of an active blank holder with adjustable spacing elements in order to increase the process reliability in the production of aluminum-plastic composites by adjusting the pressure in the workpiece plane. Similar approaches based on piezoactuators [11] in the flange area as well as electro-mechanical spacing elements [12] have already been investigated in the past.

Due to the existing boundary conditions with regard to the installation space, several hydraulic shortstroke cylinders were integrated over the holder's circumference to create a variable distance between the blank holder and the die. As a result of the active application of a pressure, the gap in the flange region can be varied over the stroke and the flow of material can be adjusted. In the present variant of the active blank holder, a common activation is achieved by a continuous hydraulic oil channel. The entire structure is shown in Figure 5.

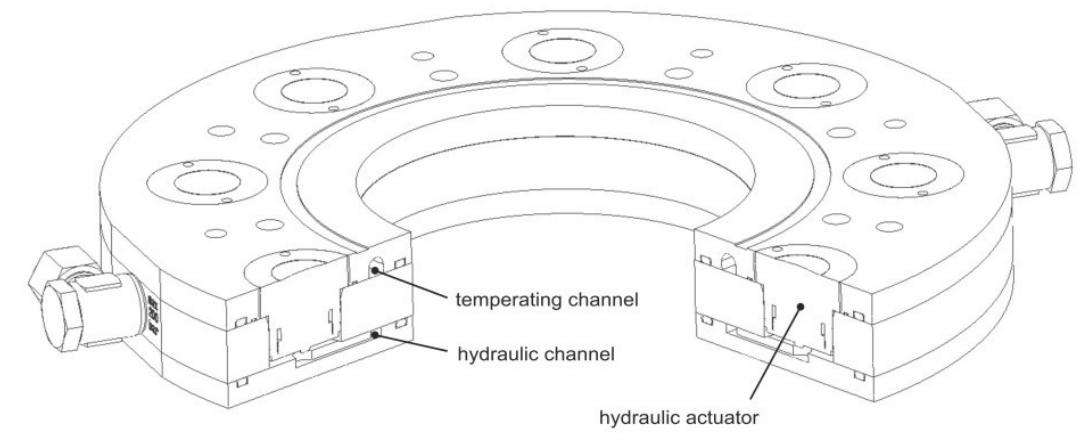

Figure 5: Schematic layout of the active blank-holder 
Before the active blank holder was applied to the complete hybrid process, further preliminary investigations had been carried out in the deep drawing stage. Since this step is largely independent from the injection process of the plastic in terms of the mode of action, this procedure can be regarded as legitimate, in particular with regard to the possibility of a resource-efficient investigation of the optimal control strategy. For this purpose, three different control variants were tested for the system pressure $p$ via the draw distance $x$, which is shown alongside the process result in Figure 6 .
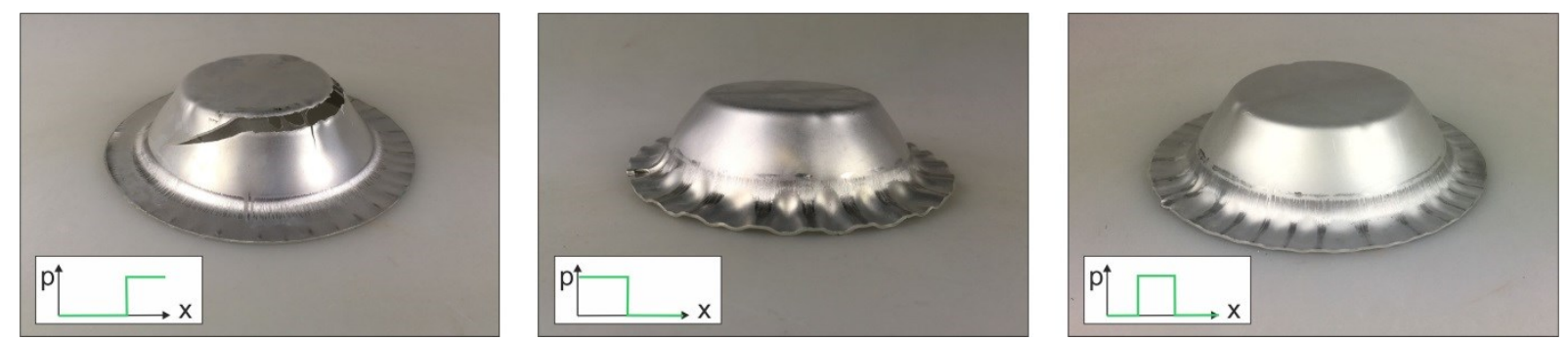

Figure 6: Control strategies and process quality impact

Obviously, multiple variations of the control signals over the draw distance lead to better component qualities in the deep-drawing step. By setting a minimum distance (low hydraulic pressure), a high surface pressure is obtained at the beginning of the drawing, which prevents initial wrinkling. As a result of the subsequent enlargement of the sheet metal holder distances, the flow of the material can now be supported. However, this is also achieved with minimal wrinkling, which can be minimized further by reducing the distance at the end of the process. These results show that the reliability for such processes can be increased in this way. Figure 7 shows a corresponding comparison of the cracked blank and the good part after using the active blank holder.

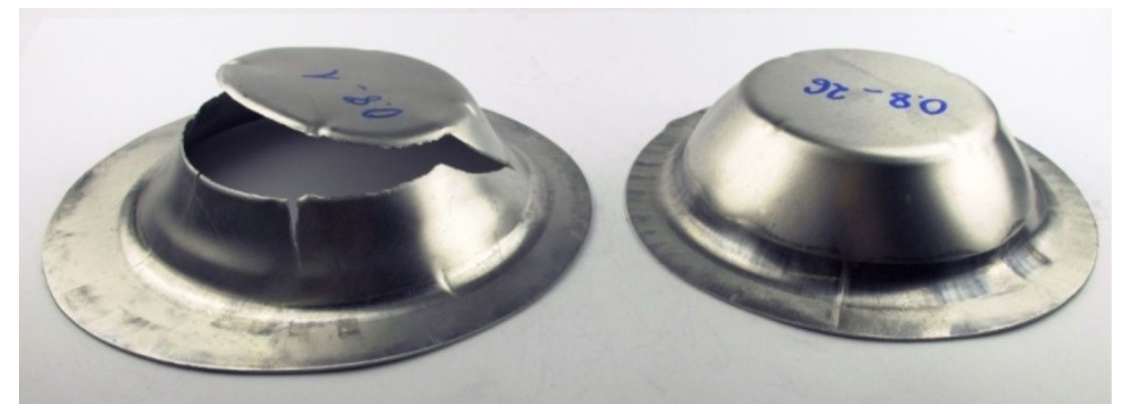

Figure 7: Comparison between a rejected and a good part

A related question considers the maximum drawing depth which could be achieved without active influence of the process. With a stepwise reduction of the drawing depth, a $5 \mathrm{~mm}$ smaller maximum drawing depth could be determined in these tests. Considering a maximum drawing depth of $30 \mathrm{~mm}$ using the active distance elements, a process limit extension by $20 \%$ could be achieved (see Figure 8).

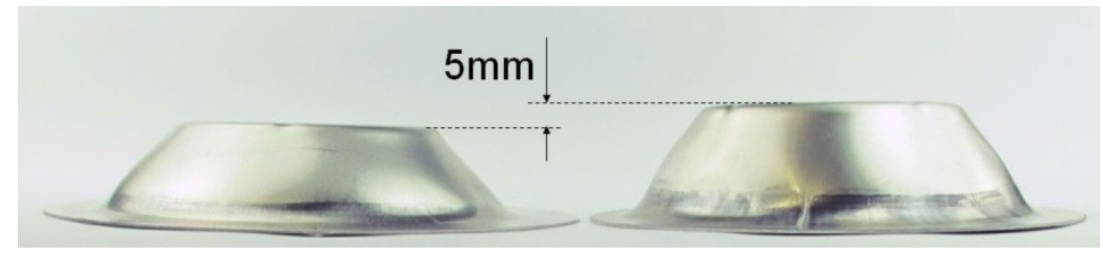

Figure 8: Extension of process limits 
After ensuring a zero-defect production of formed aluminum sheets, the active blank holder could also be applied to the complete hybrid process. This step is indispensable for the holistic evaluation of its suitability, especially since an effective media-based forming takes place through injection molding. However, the research carried out in this study showed that the shaping of the undercut does not affect the process stability. The impact of even low wrinkling in the flange area itself is clearly more critical than the result of the injection process itself. Depending on the acting blank holder force, the wrinkling increases the distance between the die and the punch in the lower dead point. This manifests in unclean injection molding. Figure 9 shows a correspondingly typical process result. The improvement of the process and optimization of the corresponding parameter selection can and should be the subject of future investigations.

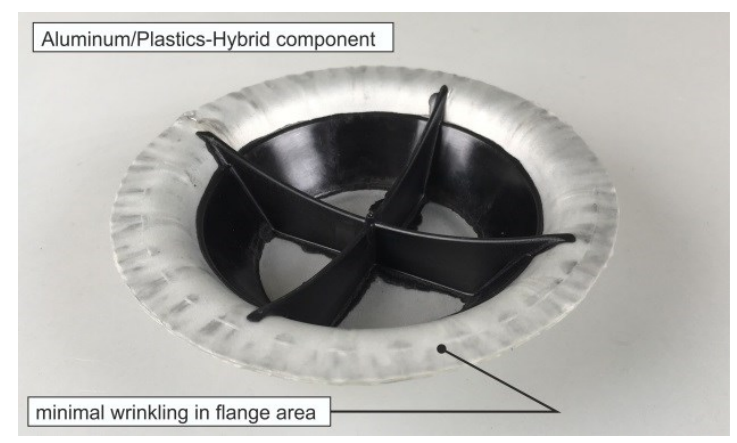

Figure 9: Aluminum/plastics hybrid component

\section{Tube based process: combination of hydroforming and injection molding}

Hybrid components made of complex shaped metallic hollow profiles and highly functional injection molding are used as cockpit cross members, front end supports and front end adapters in passenger cars, e.g. from Daimler AG [13]

The step-wise process chain, which is customarily used for sheet-metal-based metal-plastic-hybrid components, is not economical for a combination of hydroforming profiles and injection molding due to the high complexity and cost of the individual processes. Therefore, hydroforming-injection molding combines the processes hydroforming and injection molding in one tool and one plant (see Figure 10). Forming, priming and joining are carried out in one tool. The process has two stages. After the hollow profile has been inserted, it is formed by hydroforming, followed by the injection molding process (primary shaping and mating). The cavity for the injection molding is already contained in the tool. The acting liquid pressure from the previous hydroforming process into the hollow profile is held during the injection molding and prevents deformation of the hollow profile caused by the external pressure from the plastic melt. After cooling, the finished component can be removed from the forming machine.

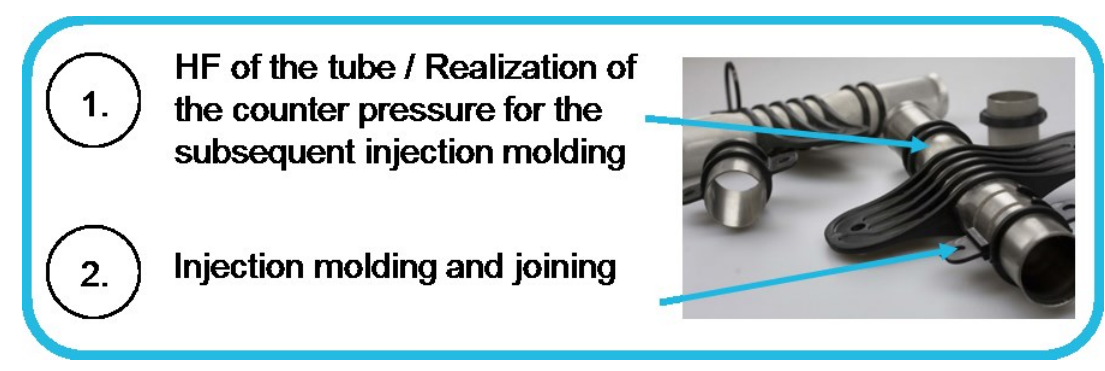

Figure 10: In-situ-process principle the hydroforming injection molding

The research work considered the usage of nitrogen as the active medium for the hydroforming process on the one hand. On the other hand, cost-intensive chemical bonding agents should be avoided by performing a surface structuring of the metallic pipes in order to increase adhesion. In 
addition, the work is aimed at developing suitable fiber-reinforced plastic tubes and adapting the process to these tubes.

\subsection{Replacement of chemical bonding agents by surface structuring}

In the case of hydroforming-injection molding with metal pipes, chemical adhesion agents are often used to increase adhesion. However, these are very expensive. As possible alternatives, various surface structures, engrailing, sandblasting and laser structuring, were tested on aluminum (EN AW6060) and steel pipes (1.4301). These were presented extensively in [14], [15] and [16]. The best results can be achieved for aluminum as well as for steel pipes with laser structuring. The used plastic was a PA6 with $60 \%$ glass-fiber-reinforcement. Table 1 shows the achieved adhesion for the laserstructured components compared to the chemical adhesion agent Vestamelt $囚 \mathrm{X} 1333$, which served as a benchmark.

Table 1: Realized bonding strengths for metal/plastic hybrid parts

\begin{tabular}{|c|c|c|c|c|c|}
\hline & \multirow{2}{*}{$\begin{array}{l}\text { materials } \\
\text { bonding agent }\end{array}$} & \multicolumn{2}{|c|}{$\begin{array}{l}\text { tube: EN AW } 6060 \\
\text { plastic: PA6 with } 60 \% \text { glass fiber }\end{array}$} & \multicolumn{2}{|c|}{$\begin{array}{l}\text { tube: } 1.4301 \\
\text { plastic: PA6 with } 60 \% \text { glass fiber }\end{array}$} \\
\hline & & Vestamelt ${ }^{\circledR}$ X1333 & $\begin{array}{l}\text { laser } \\
\text { structured }\end{array}$ & Vestamelt ${ }^{\circledR} \mathbf{X} 1333$ & $\begin{array}{l}\text { laser } \\
\text { structured }\end{array}$ \\
\hline $\begin{array}{l}\text { shear strength } \\
\text { in } \mathrm{N} / \mathrm{mm}^{2}\end{array}$ & room temperature & 19,6 & 12,3 & 19,7 & 18,1 \\
\hline \multirow{4}{*}{$\begin{array}{l}\text { tensile strength } \\
\text { in } \mathrm{N} / \mathrm{mm}^{2}\end{array}$} & room temperature & 4,7 & 1,8 & 4,1 & 1,2 \\
\hline & $80^{\circ} \mathrm{C}$ & 4,3 & 2,1 & 4,3 & 2,2 \\
\hline & $140^{\circ} \mathrm{C}$ & 3,6 & 2,3 & 4,3 & 3,0 \\
\hline & $\begin{array}{l}200^{\circ} \mathrm{C} \text { aluminum } \\
250^{\circ} \mathrm{C} \text { steel }\end{array}$ & no process possible & 2,3 & no process possible & 4,0 \\
\hline
\end{tabular}

For laser-structured steel pipes the achievable joint strengths are similar to those using Vestamelt as bonding agent. In the case of aluminum, the adhesion with the laser-structured surface was approximately $30 \%$ below the composite strength of the laser-structured steel components. Here, the component failure partially occurred in the structured surface. With an optimization of the laser structure, however, these values should be significantly improved. Both aluminum and steel tubes have to be pre-heated to achieve good tensile strengths so that the plastic melt can penetrate the surface structures well before solidification.

\subsection{Development of hydroforming injection molded components with fiber-plastic composite pipes}

In addition to hydroforming-injection molding with metal pipes, further investigations were conducted on the usage of fiber-reinforced plastic pipes. These allow for the elimination of additional adhesion agents and simplify recycling, because the same materials can be used for the pipe and the injection molding components. On the other hand, the combined process of hydroforming and injection molding places enormous demands on the pipes. In order to be able to reshape the pipes by hydroforming and to achieve a good adhesion with the injection molding component, the pipes must be pre-heated to just below the melting temperature of the matrix materials. Afterwards these soft tubes must be handled and also be gastight in the area of the cavities during the hydroforming process, which causes forming pressures up to $35 \mathrm{MPa}$. In order to achieve these aims, special pipes were developed. Tubes were chosen, which are based on an extruded short fiber-reinforced thermoplastic inner tube. This inner tube was wrapped with endless fiber-reinforced thermoplastic tapes. By using the same matrix and fiber materials for the extruded tubes and the tapes, an optimal connection of both layers could be achieved. Figure 11 shows the winding of the thermoplastic pipes as well as a finished hydroformed-injection molded component. Due to the selected pipe concept, a gas tightness of the pipes in the hydroforming injection molding process and a very good adhesion between pipe and injection molding component could be realized. 



Figure 11: Wrapping of thermoplastic tapes on plastic liners (I.h.s.), finished tube pre-product (center), Hydroforming injection molding component (r.h.s.)

\subsection{Development of a demonstrator part}

A cockpit cross-member was developed as a demonstrational part for the prototype vehicle KULAN (Polylab-Network under Fraunhofer IWU supervision). The following figure shows the part's design.

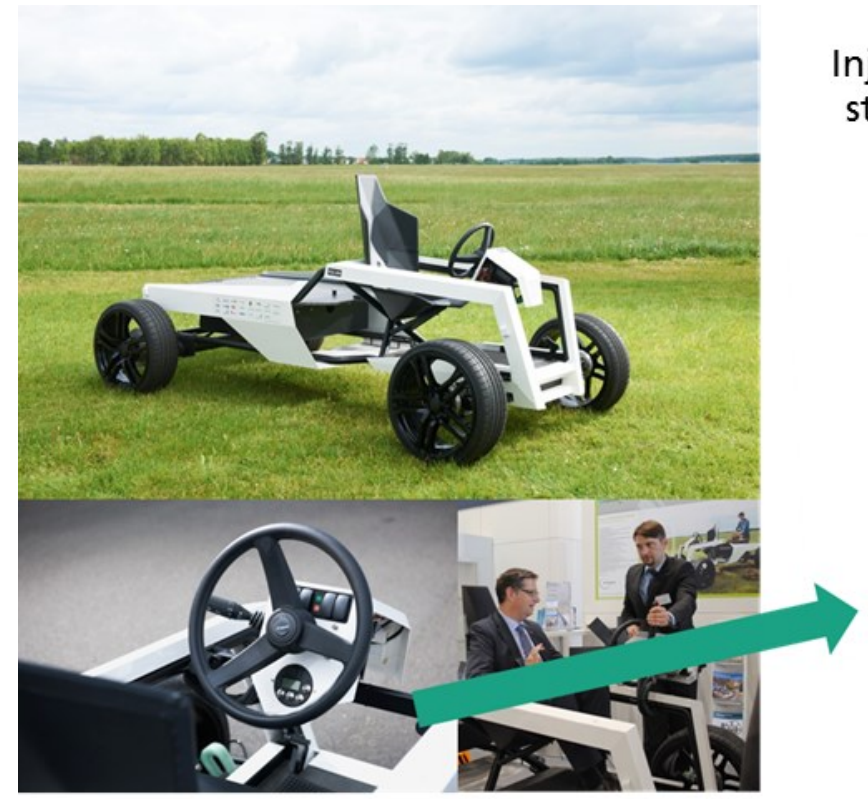

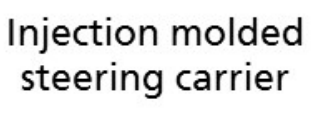

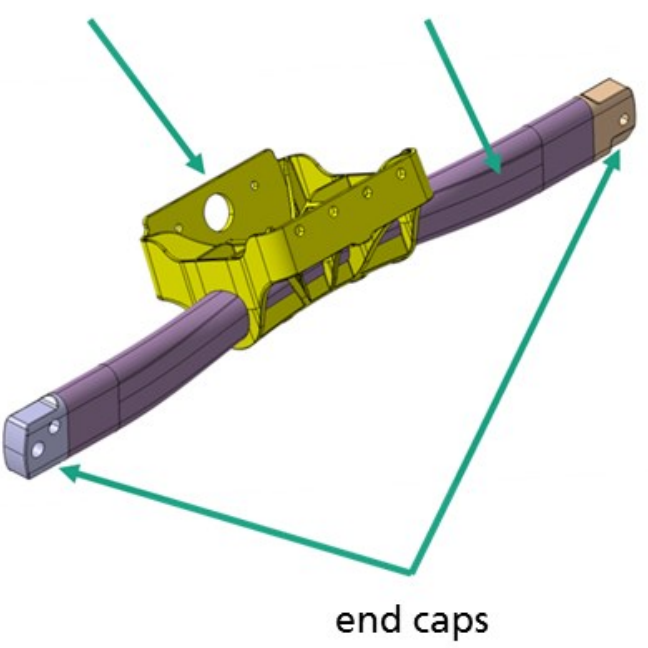

Figure 12: Demonstrational part: Cockpit cross-member for the KULAN

The component consists of an HF-shaped cross-member made of aluminum EN-AW6060 and a steering carrier, which is produced by injection molding from a glass-fiber-reinforced PA6. The end caps are separately joined after the HF/injection molding process and are used to connect the component to the vehicle structure. Due to the complexity of the aluminum part, a two-stage process with a heat treatment is necessary for realizing the HF component. The following figure shows the implemented process chain. 


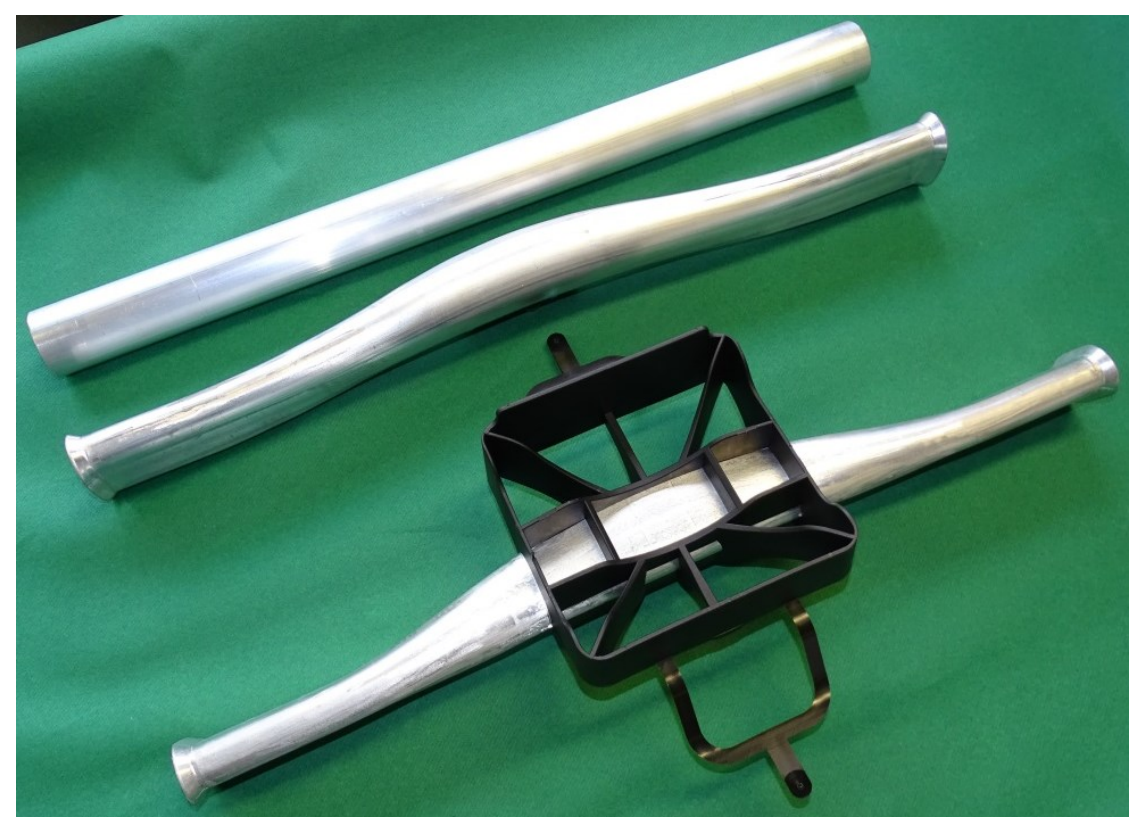

Figure 13: Production steps for the cockpit cross-member: tube preformed tube - HF/injection molded part

\section{Summary}

This paper presented two approaches of realizing a combined process for produce metal/plastichybrid structures. The operational capability was proven by experimental investigations by means of demonstrational processes. Both principles are utilisable for industrial applications, especially in the automotive industry. The first approach focused on the combination of sheet metal formed aluminum and injection molding, where the tight process limits were extended using an active blank holder. The second approach focused on the combination of hydroforming and subsequent injection molding to realize a cockpit cross-member.

\section{Acknowledgements}

This work was performed within the Federal Cluster of Excellence EXC 1075 "Merge Technologies for Multifunctional Lightweight Structures" and supported by the German Research Foundation (DFG). Financial support is gratefully acknowledged.

\section{References}

[1] Abibe, A. B.; Amancio-Filho, S.; dos Santos, J.; Hage Jr., E.: Mechanical and failure behavior of hybrid polymer-metal staked joints. Mater. and Des., 46 (2013), pp. 338-347. doi: 10.1016/j.matdes.2012.10.043

[2] Geiger M., Ehrenstein G. W.: Robuste, verkürzte Prozessketten für flächige Leichtbauteile. Tagungsband zum Berichts- und Industriekolloquium 2003 des SFB 396, p. 156.

[3] Anwendungsbeispiel: Das erste Frontend in Aluminium-Hybrid-Technik mit Durethan® BKV 30; LANXESS Deutschland GmbH, Business Unit SCP; 06.08.2007, TI 2006-047 DE.

[4] Anwendungsbeispiel: Pedallagerbock in Hybridbauweise mit Durethan® BKV 30 H2.0. LANXESS Deutschland GmbH, Business Unit SCP; 19.03.2008, TI 2007-026 DE.

[5] Endemann, U.: Leichte und wirtschaftliche Strukturen durch Kunststoff-Metall-Verbundbauweise. In: Tagungsband Mischverbindungen - Innovative Lösungen für Leichtbaukonzepte, 2002, S. 169-175. 
[6] Zorn, W.; Albert, A.; Raithel, D.; Nendel, W.; Drossel, W.-G.: Fertigung von Metall/KunststoffVerbundbauteilen: Hybridbauteile unter Verwendung von wirkmedienbasiertem Umformen herstellen. wt Werkstattstechnik online, 104(10) (2014), S. 614-619.

[7] Patent WO03/057446 A1.

[8] Bach Fr.-W., Tilmann, W., Plorin, T., Rüther, B.: Vortragsband zum Kolloquium des Graduiertenkoller 1378/1 "Herstellung, Bearbeitung und Qualifizierung hybrider Werkstoffsysteme", TU Dortmund, 10. Mai 2010.

[9] Wehmeyer, R.: Herstellung von Kunststoff/Metall-Bauteilen mit integrierter Umformung im Spritzgussprozess, Dissertation RWTH Aachen, 2012.

[10] El-Magd, E.; Abouridouane, M.: Einfluss der Umformgeschwindigkeit und -temperatur auf das Fließverhaltens metallischer Werkstoffe. Zeitschrift für Metallkunde, 94(6) (2003), pp. 729-736.

[11] Bäume, T.; Zorn, W.; Drossel, W.-G.; Rupp, G.: Step by step control of a deep drawing process with piezo-electric actuators in serial operation. Proceedings of the 4th International Conference on New Forming Technology, Glasgow, Scotland, UK, 6th to 9th August, 2015, S. 266-271.

[12] Patent: DE 10360417 B4.

[13] https://www.elringklinger.de/de/unternehmen/einblicke/kunststoff-leichtbauteile-fuer-diekarosserie, 1st August 2017.

[14] Landgrebe, D.; Müller, R.; Haase, R.; Scholz, P.; Riemer, M.; Albert, A.; Grützner, R.; Schieck, F.: Efficient manufacturing methods for hybrid metal-polymer components. ASME International Mechanical Engineering Congress and Exposition, Proceedings (IMECE), 2 (2016), ASME 2016 International Mechanical Engineering Congress and Exposition, IMECE 2016; Phoenix; United States; 11 November 2016 through 17 November 2016.

[15] Albert, A.; Drossel, W.-G.; Zorn, W.; Nendel, W.; Raithel, D.: Process combination of hydroforming and injection molding for the insitu manufacturing of metal and plastic composite structures. Mater. Sci. Forum, 825-826 (2015), pp. 522-529. doi: 10.4028/www.scientific.net/MSF.825-826.522

[16] Schieck, F.; Landgrebe, D.; Albert, A.: In-Situ manufacturing of metal-plastic Composite Structures by Process Combination of Hydroforming and Injection Molding.In: 7th International Conference on Tube Hydroforming, TUBEHYDRO 2015 : September 9-11, 2015, Xi'an, China Harbin: Harbin Institute of Technology, 2015. 